\title{
Die Musikerfamilie Bach und Hohenlohe
}

\author{
von Peter Schiffer
}

Die Familie Bach umfasste über mehrere Generationen hinweg eine Reihe von Musikern. Johann Sebastian Bach (1675-1750), der herausragende und bekannteste, ist nur einer davon. Die Familie war im 17. und 18. Jahrhundert in Thüringen und in Franken ansässig. ${ }^{1}$ Hohenlohe verfügte damals über eine Herrschaft in Thüringen. Es war die Grafschaft Gleichen mit dem Zentrum Ohrdruf, die gemeinschaftlicher Besitz der neuensteinischen Linien war. Hierzu gehörten Hohenlohe-Langenburg, Hohenlohe-Neuenstein-Neuenstein, Hohenlohe-Weikersheim, später (seit 1701) auch Hohenlohe-Ingelfingen und Hohenlohe-Kirchberg. Die hohenlohische Grafschaft Gleichen lag in der unmittelbaren Nähe der Wirkungsstätten der Musikerfamilie Bach. ${ }^{2}$

\section{Geschwister des Johann Sebastian Bach}

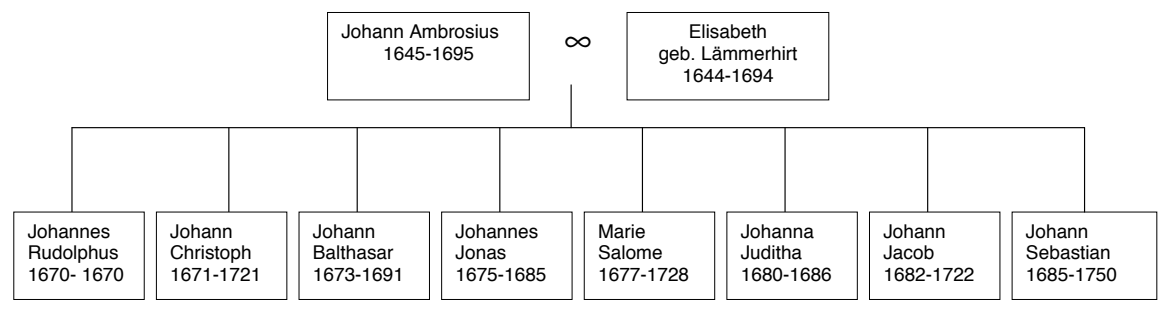

Dass Mitglieder der Familie Bach in Hohenlohe lebten, hat bereits die genealogisch orientierte Forschung festgestellt und ihr sind auch viele genealogischen Fakten bekannt. ${ }^{3}$ Hier soll es darum gehen, die Bedeutung der Musiker für das

1 Zuletzt Klaus Rüdiger Mai: Die Bachs. Eine deutsche Familie. Berlin 2013; Artikel Bach (Familie). In: Musik in Geschichte und Gegenwart (im Folgenden „MGG“) Personenteil I (1999), Sp. 1273 ff.

2 Zur Grafschaft Gleichen Adolf Fischer: Geschichte des Hauses Hohenlohe. II. Teil erste Hälfte. Stuttgart 1868, S. 40-43.

3 Gottfried Simpfendörfer: Johann Sebastian Bachs Verwandte in Württemberg. In: Südwestdeutsche Blätter für Familien- und Wappenkunde 18 (1986), S. 245-246; Kurt Hermann Frickel: Genealogie der Musikerfamilie Bach. Daten - Fakten - Hypothesen. Niederwerrn 1994; Wolfgang Caesar: Bach in Hohenlohe. In: Südwestdeutsche Blätter für Familien- und Wappenkunde 29 (2011), S. 73-79. - Zu den Quellen Rainer Trunk: Quellen zum Ohrdrufer Zweig der Musikerfamilie Bach im Hohenlohe-Zentralarchiv Neuenstein. In: WFr 86 (2002), S. 395-402 (noch ohne Berücksichti- 
hohenlohische Musikleben herauszustellen und ihre Rolle an den Höfen der Hohenlohe zu beleuchten.

\section{Johann Christoph Bach (1671-1721), Organist in Ohrdruf}

Der erste Bach, der mit Hohenlohe in Verbindung trat, war Johann Christoph, der älteste Bruder Johann Sebastians. ${ }^{4}$ Er war Schüler von Johann Pachelbel in Erfurt gewesen und trat nach kurzen Episoden in Erfurt und Arnstadt 1690 als 19-Jähriger die Stelle des Organisten an der St. Michaeliskirche in Ohrdruf an. ${ }^{5}$ Damit wurde er hohenlohischer Untertan und stand bald in Diensten des Hauses Hohenlohe. Als Grund für die Abkehr von Erfurt, seinem Geburtsort, wo er durchaus hätte bleiben können, gibt Bach später die zu dürftige Bezahlung und das zu schlechte Orgelwerk an. So gut war die Orgel in Ohrdruf aber auch nicht, um deren Verbesserung er sich zusammen mit seinem Lehrer Pachelbel lange Zeit bemühen musste. Die Bach-Familie war mit der einflussreichen Ohrdrufer Familie Eisentraut verschwägert, und es ist wahrscheinlich, dass Johann Christoph über diese Verwandten nach Ohrdruf fand und sich hier durchsetzen konnte. ${ }^{6}$

Bei seiner Einstellung als Organist an der Michaeliskirche lehnte Johann Christoph Bach eine zusätzliche Beschäftigung als Lehrer ab. Er war zu sehr Musiker, um sich anderen Aufgaben zu widmen. Sein Vorgänger im Amt war noch gleichzeitig Lehrer in der Quinta des Lyzeums gewesen. Die Einstellung war auf Dauer nicht durchzuhalten. Trotz zweier kleinerer Gehaltsaufbesserungen musste Bach im Jahr 1700 zusätzlich eine Lehrerstelle an der Lateinschule in Ohrdruf annehmen. ${ }^{7}$

Die Verbindung von Organistenamt und Lehreramt war typisch für die Zeit und begegnet auch bei anderen Mitgliedern der Familie. Die Stellung des Präzeptors, des Lehrers, nahm man in Kauf, um die Aufgaben des Organisten und andere musikalische Tätigkeiten übernehmen zu können. Mit den Schülern ließ sich aber auch musikalisch arbeiten. So wird von Aufführungen des Christspiels in Ohrdruf durch Bach und seine Schüler berichtet. ${ }^{8}$

gung der neuen Beständesignaturen des HZAN). Zum allgemeinen Hintergrund Andreas Traub: Zum Musikleben an den hohenlohischen Residenzen im 18. Jahrhundert. In: Silke Leopold / Bärbel Pelker (Hg.): Süddeutsche Hofkapellen im 18. Jahrhundert (Schriften zur südwestdeutschen Hofmusik 1), S. 117-137, Online-Publikation 2014.

4 Conrad Freyse: Die Ohrdrufer Bache in der Silhouette. Johann Sebastian Bachs ältester Bruder Johann Christoph und seine Nachkommen. Eisenstadt/Kassel 1957, S. 21-22; Konrad Küster: Der junge Bach. Stuttgart 1996, S. 65-71; Artikel Johann Christoph Bach (22). In: MGG Personenteil I, 1999, Sp. 1298.

5 La 10 CIII/1 Organist Bach, Absterben des Schulpraeceptors quintae classis Schneiders und dessen Wiederersetzung mit dem Organisten Bachen, als zugleich gewesenen Collega Sexta.

6 Küster (wie Anm. 4), S. 66 f.

7 Ebd., S. 67-71.

8 GL 35 Bü 520 und La 10 CIV/20. 
Johann Christoph starb 1721 in Ohrdruf. Einmal, 1696, hatte er Gelegenheit, sich durch eine Berufung nach Gotha zu verbessern. Er nutzte den Ruf, um bei der hohenlohischen Herrschaft eine Gehaltsaufbesserung zu erwirken. Er blieb aber in Ohrdruf, mit der Begründung: habe mich nach anruffung Gottes resolvieret lieber hier zu bleiben und mit der wenigen besoldung, nebst der addition, vorlieb zu nehmen. ${ }^{9}$ Er war also ganz zufrieden an seinem hohenlohischen Wirkungsort. Über 30 Jahre war er Organist an der Michaeliskirche. Ein Begräbniseintrag würdigt ihn als optimus artifex, also großartigen Künstler. ${ }^{10}$ Er hat komponiert, einige Cembalo-Werke, die stark an die Kompositionsweise Pachelbels erinnern, dürften von ihm stammen. ${ }^{11}$ Außerdem war Johann Christoph ein emsiger Sammler und Kopist von Werken für Tasteninstrumente aus damaliger Zeit, die ohne seine Aufzeichnung vergessen wären. ${ }^{12}$ Er ist der Stammvater des Ohrdrufer Zweiges der Musikerfamilie Bach, der uns noch beschäftigen wird.

\section{Johann Jacob Bach (1682-1722) in Ohrdruf}

1695 nahm Johann Christoph seine beiden jüngsten Brüder, die gerade Vollwaisen geworden waren, zu sich auf. Johann Jacob, der ältere von beiden, blieb nur kurz in Ohrdruf und kehrte noch im selben Jahr nach Eisenach zurück, wo er eine Stadtpfeiferlehre absolvierte. Später trat er in schwedische Dienste, zuletzt war er Kammermusiker am schwedischen Hof. Seine kurze Ohrdrufer Zeit kann nicht sehr prägend gewesen sein. ${ }^{13}$

\section{Johann Sebastian Bach (1685-1750) in Ohrdruf}

Anders war dies beim jüngsten Bruder, Johann Sebastian. ${ }^{14}$ Der war gerade zehn Jahre alt, als er sich in die Obhut seines Bruders begab. Er ging in Ohrdruf zur Schule und war ein recht guter Schüler. Johann Christoph, der gerade geheiratet hatte und eine eigene Familie versorgen musste, konnte ihn finanziell nur notdürftig unterstützen. Durch Teilnahme am Schülerchor ersparte sich Johann Sebastian das Schulgeld. Einzelne Choraufführungen brachten zusätzliche Ein-

\footnotetext{
9 Eigener Lebensbericht, abgedruckt bei Freyse: Ohrdrufer Bache (wie Anm. 4), S. 83.

10 Zitiert nach Freyse: Ohrdrufer Bache (wie Anm. 4), S. 21.

11 Artikel Johann Christoph Bach (22), in: MGG Personenteil I, 1999, Sp. 1298.

12 Zum „Andreas-Bach-Buch“ und zur „Möllerschen Handschrift“, die unter seiner Hand entstanden, Hans-Joachim Schulze: Studien zur Bach-Überlieferung im 18. Jahrhundert. Leipzig 1984, S. 30-56, besonders S. $53 \mathrm{f}$.

13 Artikel Johann Jacob Bach (23). In: MGG Personenteil I, 1999, Sp. 1303.

14 Über Johann Sebastian Bach in Ohrdruf Küster: Der junge Bach (wie Anm. 4), S. 62-81; Karl Geiringer: Die Musikerfamilie Bach. Leben und Wirken in drei Jahrhunderten. München 1958, S. 135-138 und Werner Breig: Artikel „Johann Sebastian Bach“. In MGG Personenteil 1 (1999), Sp. $1397 \mathrm{ff}$.
} 
künfte. Nach eigenem Zeugnis verfügte Johann Sebastian über eine ungemein schöne Sopranstimme. ${ }^{15}$

Die Ohrdrufer Zeit des jungen Bach, immerhin fünf Jahre, war für dessen musikalische Entwicklung prägend. Bei seinem Bruder lernte er die Grundlagen des Klavier- und Orgelspiels. ${ }^{16}$ Eine Anekdote berichtet, der junge Bach habe entgegen dem Verbot des Bruders aus dessen verschlossenem Schrank Noten von Froberger, Kerll und Pachelbel gefingert und diese mit ungeheurem Bildungshunger bei Nacht im Mondlicht für sich abgeschrieben. Der Bruder war Schüler Pachelbels, die fraglichen Noten waren Erinnerungen an seine Lehrjahre und deshalb gut verschlossen. Die genannten Komponisten verkörpern die süd- und mitteldeutsche Orgeltradition, mit der Johann Sebastian Bach in der Ohrdrufer Zeit vertraut wurde. Über das Erlernen des musikalischen Rüstzeuges hinaus beeinflusste der ältere Bruder die Berufsorientierung. Durch das persönliche Vorbild des älteren Bruders lernte Johann Sebastian die Tätigkeit eines Organisten konkret kennen. Diesen Berufsweg schlug er später ein. ${ }^{17}$ Johann Sebastian Bach hat in der Ohrdrufer Zeit komponiert, einige Orgel- und Klavierstücke können der Zeit vor 1700 zugeordnet werden. ${ }^{18}$ Im Jahr 1700 verließ er aus finanziellen Gründen Ohrdruf. Eine kostenfreie Schulstelle stand hier nicht mehr zur Verfügung. Er setzte, unter Vermittlung des Ohrdrufer Kantors Herda, seine schulische Ausbildung in Lüneburg fort.

\section{Bach in Weikersheim}

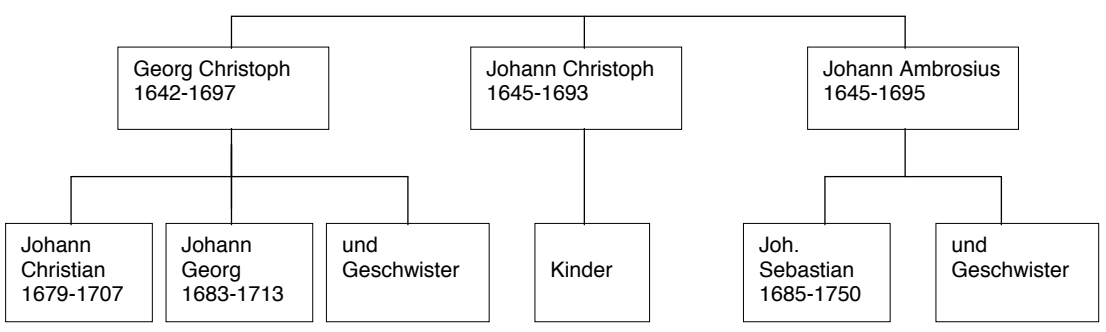

15 Zitiert nach Küster (wie Anm. 4), S. 77.

16 Geiringer (wie Anm. 14), S. 136; Küster (wie Anm. 4), S. 77-81.

17 Küster (wie Anm. 4), S. $77 \mathrm{f}$.

18 Aus der Ohrdrufer Zeit Bachs könnten laut Wolfgang Schmieder: Thematisch-systematisches Verzeichnis der Werke Johann Sebastian Bachs. Leipzig 1986, S. 649 stammen: BWV 945 Fuge e-Moll, BWV 743 Choralvorspiel „Ach, was ist doch unser Leben“, BWV 749 Choralvorspiel „Herr Jesu Christ, dich zu uns wend“, BWV 750 Choralvorspiel „Herr Jesu Christ, meins Leben Licht“; BWV 751 Choralvorspiel „In dulci jubilo“, BWV 755 Choralvorspiel „Nun freut euch, lieben Christen g'mein“, BWV 756 Choralvorspiel „Nun ruhen alle Wälder“, BWV 757 Choralvorspiel „O Herre Gott, dein göttlichs Wort“ und BWV 762 Choralvorspiel „Vater unser im Himmelreich“. Vielleicht stammen noch aus der Ohrdrufer Zeit BWV 766 Partite diverse über „Christ der du bist der helle Tag“, BWV 767 Partite diverse über „O Gott, du frommer Gott“ und BWV 768 Variationen I, II, IV und X aus den Partite diverse über „Sei gegrüßet, Jesu gütig“. 


\section{Johann Christian Bach (1679-1707) in Weikersheim}

Die nächste Verbindung der Musikerfamilie mit Hohenlohe erfolgte nur wenig später - 1703. Ein Vetter Johann Sebastian Bachs, Johann Christian Bach, bewarb sich um eine Stelle in Weikersheim. Er war Sohn des in Schweinfurt wirkenden Georg Christoph Bach, des Bruders von Ambrosius Bach, des Vaters von Johann Sebastian Bach. Georg Christoph Bach gehörte der fränkischen Linie der Familie an. ${ }^{19}$ Die Bewerbung erfolgte zu einer Zeit, als absehbar war, dass die Herrschaft Hohenlohe-Oehringen zwischen den Brüdern Carl Ludwig und Johann Friedrich II. geteilt würde. 1702 war deren Vater Johann Friedrich I. von Hohenlohe-Oehringen gestorben. Und es war schon damals wahrscheinlich, dass Weikersheim für einen der Brüder Residenz werden würde. ${ }^{20}$

In Neuenstein hatte Johann Christian sich beim Hofprediger und Superintendent Christian Höber einer zweitägigen Probe in Musik wie in Schuldingen unterzogen. Hier legte er am 28. August seine in Latein abgefasste Bewerbung vor. Über die musikalischen Fähigkeiten und die Befähigung in Schuldingen gutachtete Höber und befand, daß er [Bach] in beyden von solcher Capacität ist, als man zu Weikersheim nötig hat. Weil er fürchtete, Bach könne nach Schweinfurt zurückgehen, votierte er gegenüber dem Konsistorium für eine schnelle Entscheidung. Man dürfte schwerlich einen geschickteren zu dieser Funktion und schlechten Besoldung bekommen. ${ }^{21}$ Am gleichen Tag informierte das Konsistorium das Amt Weikersheim über die positive Entscheidung der Brüder Carl Ludwig und Johann Friedrich von Hohenlohe-Oehringen. Die Stelle eines Collaboratoren, also eines Lehrergehilfen, war seit längerem in Weikersheim vakant und es gab auch weitere Bewerbungen. Allein durch sein Können, vor allem sein musikalisches Können, hat sich der Theologiestudent Johann Christian Bach durchgesetzt. Die Bewerbungsakten belegen, daß er nicht allein ratione studiorum hier gnedigst umb qualifizieret, sondern auch in Musicis, als im Singen, Geigen und tractiern des General Basses [solide Kenntnisse besitze] und dem Orgelwerk eine hochstehende Wissenschaft aufweise. ${ }^{22}$ Leise bezweifelte das Konsistorium nur die Eignung als Lehrer, weshalb Bach zunächst probeweise eingestellt wurde. Bach bewährte sich auch in der pädagogischen Praxis und wurde 1706 zum Präzeptor in Weikersheim befördert. ${ }^{23}$

19 Frickel (wie Anm. 3), S. 26 f.; Artikel Georg Christoph Bach (10). In: MGG Personenteil I, 1999, Sp. 1291.

20 Stammtafel 5 des Hauses Hohenlohe. Öhringen 1939 Über Karl Ludwig von Hohenlohe-Weikersheim Adolf Fischer: Geschichte des Hauses Hohenlohe. II. Teil zweite Hälfte. Stuttgart 1871, S. 233-249.

21 We 41 Schubl. 34 Fasz. 60a, Zitate aus Qu. 12. Zur Bewerbung Johann Christian Bachs in Weikersheim Joachim Kremer: „Von dem Musikerbiographik des frühen 18. Jahrhunderts. Neumünster 2014, S. $315 \mathrm{ff}$. Ihm geht es primär um Bewerbungsschreiben und -unterlagen als Quellen zur Musikerbiographik.

22 We 41 Schubl. 34 Fasz. 60a, Zitat aus Qu. 9.

23 Er wird in We 41 Schubl. 34 Nr. 62 e von 1707 als Präzeptor bezeichnet. 
Johann Christian Bach heiratet 1704 die Tochter des hohenlohischen Registrators und Stadtschreibers von Weikersheim, worin seine gesellschaftliche Akzeptanz in der neuen Heimat deutlich wird. Gleichzeitig mit seiner Einstellung war eine Renovierung, wenn nicht gar Erneuerung der Weikersheimer Orgel ins Auge gefasst worden. Die Grafenbrüder in Öhringen übten die Herrschaft noch gemeinschaftlich aus, aber eine Teilung, die 1708 durchgeführt wurde, war abzusehen. So wollte man auch in musikalischer Hinsicht Weikersheim als Residenz ausstatten. Im April 1705 rügte Johann Christian Bach, unterstützt vom Präzeptor Christian Ludwig Saager und dem Weikersheimer Amtmann Gebhard, den schlechten Zustand der Orgel in Weikersheim und regte an, ein Gutachten durch den Orgelmacher von Neuenstein, Otto Reinhard Metzenius, erstellen zu lassen. ${ }^{24}$ Metzenius begutachtete daraufhin die Orgel. ${ }^{25}$ Die Regierung in Öhringen entschied sich für einen Neubau - wenn das Geld dafür da sei. Johann Christian Bach unterstützte die Orgelerneuerung tatkräftig, doch erlebt hat er sie nicht mehr. Schon 1707 starb er im Alter von nur 28 Jahren.

\section{Johann Georg Bach (1683-1713) in Weikersheim}

Johann Christian Bachs jüngerer Bruder Johann Georg ${ }^{26}$ kam 1705 nach Weikersheim und hat hier dem Amtmann Gebhard bei der Schreibarbeit und bei der Erziehung der Kinder geholfen. In Forchtenberg kümmerte er sich um die vakante Lehrerstelle. ${ }^{27}$ Nach dem Tod seines Bruders bewarb er sich umgehend bei den Grafenbrüdern in Öhringen auf dessen Stelle, wobei er engagierte Empfehlungen des Amtmanns, des Pfarrers von Weikersheim sowie auch von Bürgern der Stadt beilegen konnte. Er verfügte also in Weikersheim bereits über einen beachtlichen Rückhalt. Das Konsistorium in Öhringen setzte ihn im Juli 1707 in die Stelle des Präzeptors ein. In seinem Bewerbungsschreiben hatte Johann Georg als sein Lebensziel angegeben, meinem Gott, dermahleins in einer Schule der Jugend und bei dem Gottesdienst der Instrumentalmusique [...] zum besten $z u$ dienen. ${ }^{28}$ Viel Zeit blieb ihm nicht. 1713 starb er, nur sechs Jahre hat er in Weikersheim als Lehrer und Organist wirken können. Von seinen Kindern lebte nur eine Tochter länger. Sie heiratete 1732 den Weikersheimer Kantor Wagner, worin sich noch einmal die Vorliebe der Familie für die Musik zeigt. Der Sohn seines Bruders, Johannes Paul Ludwig, zog 1738 nach Schweinfurt. Weitere

24 We 41 Schubl. 34 Fasz 61 Qu. 2.

25 Zur Neuanschaffung einer Orgel in Weikersheim We 41 Schubl. 34 Fasz 61, über Metzenius Hans F. Pfeiffer: Otto Reinhard Metzenius - der Orgelmacher von Hall. In: WFr 87 (2003). S. 61121, zur Neuensteiner Zeit des Orgelbauers S. 92 ff.

26 Frickel (wie Anm. 3), S. 26 und 43. Vgl. hierzu auch Kremer (wie Anm. 21), S. 323 ff.

27 We 41 Schubl. 34 Nr. 62 e Qu. 1-3.

28 We 41 Schubl. 34 Fasz 62 e Qu. 3. 


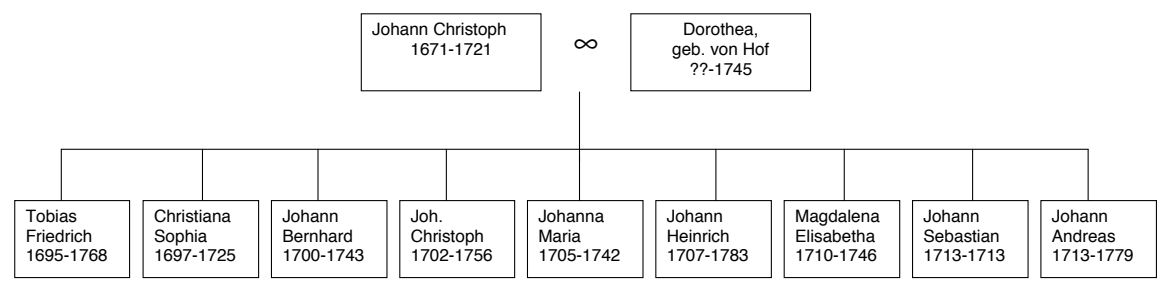

Weikersheimer Mitglieder der Musikerfamilie Bach unter den Nachfahren der beiden Brüder gab es also nicht. ${ }^{29}$

\section{Tobias Friedrich Bach (1695-1768) in Ohrdruf}

Zurück zu dem Ohrdrufer Zweig der Familie Bach, zu den Söhnen des Johann Christoph Bach. Bei dessen Tod 1721 waren sieben Kinder unversorgt. Für den ältesten Sohn, Tobias Friedrich, ${ }^{30}$ konnte der Vater noch Vorkehrungen treffen. Als 1714 eine zweite Kirche in Ohrdruf eingerichtet wurde, die Trinitatis-Kirche, erhielt sein Sohn das Organistenamt. Tobias Friedrich Bach blieb aber nur drei Jahre (bis 1717) in Ohrdruf. Anschließend wirkte er in der Braunschweiger Gegend und später in der Nähe von Weimar.

\section{Johann Bernhard Bach (1700-1743) in Ohrdruf}

Der zweitälteste Sohn, Johann Bernhard, ${ }^{31}$ erhielt 1721 auf Initiative des Ohrdrufer Stadtrates sowie unter Zustimmung der Regierungen in Öhringen und Ingelfingen die Stelle des Vaters, also das Organistenamt an der Ohrdrufer Michaeliskirche. Hiervon wurden die schulischen Verpflichtungen und die damit verbundenen Einnahmen abgetrennt. ${ }^{32}$

29 Frickel (wie Anm. 3), S. 43 ff.

30 Freyse (wie Anm. 4), S. 22-23; Artikel Tobias Friedrich Bach (40). In: MGG Personenteil I, 1999, Sp. 1309.

31 Freyse (wie Anm. 4), S. 28-34, S. 85; Artikel Johann Bernhard Bach (41). In: MGG Personenteil I, 1999, Sp. 1295.

32 GL 35 Bü 574 Qu 2, Besoldung des Organisten Johann Bernhard Bach zu Ohrdruf, 1733-1741; GL 35 Bü 595, Besoldungserhöhung für den Organist Bach, 1733; La 10 I 7 und Besoldungserhöhung für den Organist Bach, 1736, La 10 K 28/3. 
Johann Bernhard war Schüler seines Onkels Johann Sebastian Bach. ${ }^{33}$ Zwischen 1715 und 1719 hat er bei ihm in Weimar und Köthen gelebt und u. a. als Notenkopist gewirkt. Das Abschreiben und handschriftliche Vervielfältigen von Noten war im Hause Bach in größerem Ausmaß erforderlich. Am 29. Mai 1731 heiratete Johann Bernhard die Langenburgerin Anna Christina Roth. ${ }^{34}$ Er muss intensivere Kontakte zu der hohenlohischen Residenz gehabt haben, über die jedoch Einzelheiten nicht bekannt sind. Auch Johann Bernhard hat komponiert, Werke für Tasteninstrumente sind von ihm bekannt. Er hatte Bedeutung vor allem als Notensammler und -tradierer und war der maßgebliche Schreiber des AndreasBach-Buches und der Möllerschen Handschrift. ${ }^{35}$ Zusammen mit seinen Brüdern führte er in Ohrdruf 1734 und 1736 Kantaten auf, darunter auch mindestens eine von seinem bekannten Onkel. ${ }^{36}$

\section{Johann Christoph Bach (1702-1756)}

Der dritte Sohn, der wie der Vater Johann Christoph ${ }^{37}$ hieß, hatte 1723 in Jena ein Jura-Studium begonnen. Durch zahlreiche Bittbriefe bei den Grafen von Hohenlohe erreichte er und seine Mutter eine kräftige Unterstützung seines Studiums aus der gemeinschaftlich langenburgischen Stipendien- und Schulcollectur. ${ }^{38}$ Nach Beendigung des Studiums 1726 kehrte der studiosus iuris ${ }^{39}$ Johann Christoph nach Ohrdruf zurück. Hier unterstützte er den kränkelnden Kantor Elias Herda. Als dieser im Frühjahr 1728 starb, bewarb er sich um die Nachfolge. Es gab mehrere Konkurrenten. Der Ohrdrufer Rat votierte im Juni für Bach, im Mai schon hatte sich der Graf von Hohenlohe-Ingelfingen auf ihn festgelegt. Für Bach sprachen eindeutig die wegen seines verstorbenen Vatters gehabten Meriten und seiner armen Mutter zu einigem Trost, und das Bemühen, ihm in regard seines Vatters undt der bachischen Familie außer allem Zweifel favorable zu sein. Die Öhringer Regierung blieb zögerlich. Mitbewerber war unter anderem der Kantor des nahegelegenen Wechmar, der sich auf Zusagen berief, später in Ohrdruf eingestellt zu werden. Möglich war ein Tausch derart, dass Bach nach Wechmar ging und der Wechmarer die Stelle in Ohrdruf übernahm. Die Behör-

33 Hans Löffler: „Bache“ bei Sebastian Bach. In: Bach-Jahrbuch 1949-1950. Leipzig 1950, S. 106-124, besonders 108-110.

34 Freyse (wie Anm. 4), S. 31.

35 Ebd., S. 28; Artikel Johann Bernhard Bach (41). In: MGG Personenteil I, 1999, Sp. 1295.

36 Ebd., Sp. 1295.

37 Freyse (wie Anm. 4), S. 34-65, S. 86; Artikel Johann Christoph Bach (42). In: MGG Personenteil I, 1999, Sp. 1299.

38 Stipendiatsgesuche des Johann Christoph Bach 1723-1724, La 10 D 28/1 und GL 35 Bü 719.

39 Schreiben des Konsistoriums in Ohrdruf an die Regierung Öhringen vom 30. Mai 1728, in GL 35 Bü 589. 
den in Öhringen und Ingelfingen konnten keine Einwilligung Bachs bewirken, der sich in Ohrdruf schon unter Herda eingearbeitet hatte und die Zusammenarbeit mit seinem Bruder Johann Bernhard anstrebte. Kantoren- und Organistenamt wären ja dann in Bach'schen Händen. Nun wurde auch das Ohrdrufer Konrektorat vakant, also eine Lehrerstelle in leitender Funktion, und beide hohenlohischen Linien einigten sich auch darauf, diese mit Bach zu besetzen. Der Kompromißversuch scheiterte. Ende August wurde die Kantorfrage in Ohrdruf entschieden - zugunsten Bachs.

Die Akten über die Stellenbesetzung in Ohrdruf 1728 lassen die Gründe für die Entscheidung erkennen. Die Verdienste des Vaters, der über 30 Jahre in Ohrdruf gewirkt hatte, wurden vom Bewerber, vom Ohrdrufer Rat wie vom Ingelfinger Grafen und auch seitens der Öhringer Verwaltung als maßgeblich hervorgehoben. Als weiteres kam die Not der Witwe mit ihren zahlreichen noch zu versorgenden Kindern hinzu. In der Besetzung des Ohrdrufer Kantorats wird die posthume Beliebtheit Johann Christoph Bachs, des Vaters, in Ohrdruf und im restlichen Hohenlohe deutlich. ${ }^{40}$

Wie sein Bruder heiratete Johann Christoph am 24. November 1731 eine Langenburgerin, nämlich Johanna Christina Sophia Meyer, Tochter des Kanzleisekretärs Georg Heinrich Meyer. ${ }^{41}$ Auch er muss Beziehungen und Verbindungen nach Langenburg gehabt haben. Einzelheiten bleiben unbekannt.

Aufgabe des Kantors war die Einstudierung von Choraufführungen der Schulknaben. Die Zusammenarbeit mit dem Ohrdrufer Organisten, dem Bruder Johann Bernhard, ergab sich zwangsläufig. Gemeinsam mit seinen Brüdern Johann Bernhard und Johann Heinrich führte Bach Kantaten auf. Er wirkte an der Komposition eines kantatenähnlichen Stückes anlässlich der Verheiratung des Grafen Ludwig Friedrich Karl zu Hohenlohe-Oehringen mit. ${ }^{42}$ Eine Beschwerde über den Kantor lässt dessen Engagement gut erkennen. Superintendent Rothmaler zu Ohrdruf berichtete am 15. Januar 1737 an die Grafen von Hohenlohe über greuliche Ärgernisse und Turbationes des öffentl. Gottesdienstes durch Kantor Bach. Er habe einen Musikantenchor statt eines Schülerchores eingerichtet, was zu viel Raum einnehme. Der Bruder des Kantors, der Organist Bach, mache mit ihm eine Sache, daß er nicht nur die Pauken öffterer als sonst geschehen, traktieret, und solchen Lärm damit vorführet, als sollte eine opera gespielet werden. ${ }^{43}$

40 Bewerbung des Johann Christoph Bach um die Stelle des Kantorats am Gymnasium zu Ohrdruf (nach Absterben des Kantors Elias Herda), 1728, GL 35 Bü 589, hieraus die beiden Zitate; Besetzung der Stelle des verstorbenen Kantors Herda mit Johann Christoph Bach, 1728, La 10 F 27 und GL 35 Bü 590.

41 Freyse (wie Anm. 4), S. 34-37.

42 Cantata anlässlich der Heirat Graf Ludwig Friedrich Carls zu Hohenlohe-Oehringen mit Sophia Amalia Carolina 1749, Text in PA 31/1/7-1.

43 La 10 K 50 Qu 1. 


\section{Johann Heinrich Bach (1707-1783) in Öhringen}

Der vierte Sohn des Johann Christoph Bach war Johann Heinrich. ${ }^{44}$ Spätestens 1735 wurde er Organist und Präzeptor in Öhringen. Kurz vorher war in der Stiftskirche eine neue Orgel gebaut worden. ${ }^{45}$

Seine Bewerbung, die als Öhringer Angelegenheit Sache des Gesamthauses Hohenlohe war, ist leider nicht aktenmäßig dokumentiert. Sein Orgelspiel wird gerühmt, sein Zeitgenosse Wibel bezeichnet ihn als ein geschickter Musikus. ${ }^{46}$ Mit seinem Vorgänger im Amt, Johann Joachim Planck, dessen Adjunkt er zunächst war, stand er in Streit. ${ }^{47}$

Auch Johann Heinrich hat bei seinem Onkel Johann Sebastian gelernt und gewirkt ${ }^{48}$ und zwar zwischen 1724 und Frühjahr 1728 in Leipzig, wo er Schüler an der Thomasschule war. Er ist wie Johann Bernhard als Notenkopist in der Umgebung Bachs nachweisbar. Anlässlich der Hochzeit Ludwig Friedrich Carls von Hohenlohe-Oehringen 1749 komponierte er zusammen mit seinem Bruder Johann Christoph eine Huldigungskantate, deren Text erhalten ist, aber nicht die Noten. Der Titel lautete: Cantata, welche nach glücklich vollzogenem Beylager zu Hildburghausen des hochgebohrnen Grafen und Herrn, Herrn Ludwig Friedrich Carl, Grafen von Hohenlohe und Gleichen, Herrn zu Langenburg und Cranichfeld mit der durchlauchtigsten Fürstin und Frauen, Frau Sophia Amalia Carolina, gebohrnen Herzogin zu Sachsen, Jülich, Cleve und Berg, auch Engern und Westfalen, Landgräfin in Thüringen, Markgräfin zu Meißen, gefürstete Gräfin zu Henneberg, Gräfin zu Marck und Ravensberg, Frau zu Ravenstein etc. bei der den Septembr. 1749 erfolgten hoch-vergnügten Heimführung in Öhringen untertänigst überreicht und bei einer Tafel-Musik abgesungen worden, von Johann Christoph Bach und Johann Heinrich Bach. ${ }^{49}$ Während der Feierlichkeiten schlug Johann Heinrich vom Turm aus die Pauken. ${ }^{50}$ Damit übernahmen beide Brüder die Aufgaben eines Hofkomponisten, indem sie für ein wichtiges Hoffest die feierliche Musik schrieben.

Aus den Besoldungsakten geht hervor, dass auch in Öhringen das Kurrendesingen durch den Schülerchor Weihnachten und Neujahr üblich war, wofür Bach

44 Ernst Seeger: Johann Heinrich Bach. Lehrer und Kantor in Öhringen. In: Hohenloher Chronik 3 , Nr. 9 (1955), S. 4; Freyse (wie Anm. 4), S. 67-69; Simpfendörfer (wie Anm. 3), S. 245 f.; Artikel Johann Heinrich Bach (43). In: MGG Personenteil I, 1999, Sp. 1303; Caesar (wie Anm. 3), S. 76.

45 Ernst Seeger: Aus dem musikalischen Leben im alten Öhringen. In:Öhringen. Stadt und Stift. Öhringen 1988, S. 178-180, hier S. 179.

46 Johann Christian Wibel: Hohenlohische Kyrchen- und Reformations-Historie, 1. Teil. Onolzbach 1752. S. 588 .

47 PA 104/5/5 Qu 159-170.

48 Löfler (wie Anm. 33), S. $111 \mathrm{f}$.

49 PA $31 / 1 / 7-1$ und 2.

50 PA 31/1/7-1. 
einen, seiner Meinung nach zu geringen Betrag erhielt. Auch bei Beerdigungen und Hochzeiten war der Schülerchor unter Leitung Bachs aktiv. ${ }^{51}$

Obwohl sich Johann Heinrich 1743 auf die Kantorstelle im thüringischen Wechmar bewarb, ${ }^{52}$ blieb er zeit seines Lebens in Öhringen, wo er erst 1783 starb. Über drei Jahrzehnte hindurch, bis zu seiner Pensionierung, wirkte er in Öhringen als Organist und Lehrer. Damit hat er länger in Öhringen als in seiner thüringischen Heimat gelebt und gewirkt. Er war zweimal verheiratet, seine erste Frau Marie Susanne Renner war die Tochter eines Gastwirtes aus Neuenstadt am Kocher. Die 1735 geschlossene Ehe blieb kinderlos. 1749 heiratete Bach die Pfarrerstochter Maria Christine Brinkmann aus Gnadental. Von den elf Kindern überlebten nur zwei Töchter. Seine Familie lebte nur in weiblicher Linie fort. Eine Tochter heiratete den Nachfolger Bachs, den Präzeptor Gottfried Ernst Sallmann, der ein tüchtiger Komponist gewesen sein soll. ${ }^{53}$

\section{Johann Andreas Bach (1713-1779)}

Johann Andreas Bach ${ }^{54}$ war der jüngste Sohn von Johann Christoph. Nachdem er als Oboist im Gothaischen Dragonerregiment am Polnischen Erbfolgekrieg teilgenommen hatte, trat er 1737 als Tafeldecker in langenburgische Dienste. Vier Jahre lang war er in der engsten Umgebung des Grafen von Hohenlohe-Langenburg tätig. Dass er sich daneben musikalisch betätigte, ist zu vermuten. Musikalische Tätigkeiten werden in den knappen Quellen nicht direkt angesprochen, es ist nur von einer Anstellung als Lakai bzw. Tafeldecker die Rede. Da an den hohenlohischen Höfen Musiker häufig aus der Dienerschaft rekrutiert wurden, ist davon auszugehen, dass man sich der Oboen- bzw. Orgelkenntnisse Bachs bei musikalischen Aufführungen, über die sich kaum Quellen finden, am Hof bediente. Johann Andreas Bach wird jedenfalls 1741 ausdrücklich als Musicus bezeichnet. $^{55}$

1741 endete seine Beschäftigung durch abrupte Flucht. Er hatte eine Dienerin geschwängert und ihm drohte auch deshalb eine hohe Strafe, weil diese als Vollwaise dem besonderen Schutz des Langenburgers unterstand. Johann Andreas floh zu seinem Bruder Johann Heinrich nach Öhringen. In einem Brief an seine Geliebte entwickelt Johann Andreas fantastische Berufsperspektiven - als Orga-

51 PA 104/5/5 Qu 166 und 167. Seeger (wie Anm. 45), S. 9; PA 104/5/5 Qu 166 und 167.

52 Bewerbung des Johann Heinrich Bach zu Öhringen um die Kantorstelle zu Wechmar, 1743, GL

35 Bü 611 .

53 Seeger (wie Anm. 45), S. 9; Freyse (wie Anm. 4), S. 69.

54 Freyse (wie Anm. 4), S. 71-81, 87; Artikel Johann Andreas Bach (44). In: MGG Personenteil I, 1999, Sp. 1294.

55 Über die Zeit als Tafeldecker in Langenburg La 35 Regierung II Bü 841. - Kirchensaller Taufbuch, Eintrag vom 31. Mai 1741. - MGG I (1949), S. 915 vermutet als „Hofmusiker“. Eine Nebenbeschäftigung als Musiker in Langenburg nimmt auch Hans-Joachim Schulze: Studien zur BachÜberlieferung (wie Anm. 12), S. 34 an. 
nist in Heilbronn, in Dinkelsbühl und zur Not als Oboist in württembergischen Militärdiensten wollte er wirken. ${ }^{56}$ Der Graf von Hohenlohe-Langenburg ließ in Ohrdruf das Vermögen Bachs schätzen, ohne es aber einziehen zu können. Da hatte Hohenlohe-Oehringen ein Wort mitzureden. Außerdem war das Vermögen nicht so beträchtlich, wenn man das elterliche Haus auf alle Erben einschließlich der Witwe aufteilte. ${ }^{57}$

Durch Vermittlung der Kanzlei Öhringen konnte die peinliche Situation gemeistert werden. Die Heirat Bachs mit der Langenburgerin, eine geborene Hofmann, fand in aller Stille und unbemerkt von der Langenburgischen Gesellschaft in Gnadental statt. Das Kind, Maria Sophia Catharina, kam am 30. Mai 1741 im abgelegenen Kirchensall zur Welt..$^{58}$

Johann Andreas ging nach Ohrdruf zurück, in dessen Umgebung, in Werningshausen, ihm der Graf von Hohenlohe-Langenburg eine Organistenstelle in Aussicht gestellt hatte. Diese besetzte jedoch der Ohrdrufer Superintendent eigenmächtig anderweitig. ${ }^{59} 1742$ erhielt Bach endlich eine Stelle, und zwar als Lehrer in der Ohrdrufer Vorstadt, ${ }^{60}$ womit die Aufgabe als Organist an der Trinitatiskirche verbunden war, an der schon sein ältester Bruder gewirkt hatte. Als im nächsten Jahr durch den Tod seines Bruders die Organistenstelle an der Michaeliskirche vakant wurde, trat er dort die Nachfolge an. Im November 1743 schrieb Johann Andreas Bach an die Grafen von Hohenlohe-Oehringen, Hohenlohe-Langenburg, Hohenlohe-Kirchberg und Hohenlohe-Ingelfingen einen Brief, in dem er sich vor die mir erzeigte hohe Gnade und väterliche Vorsorge bei der Besetzung der Organistenstelle in Ohrdruf bedankte. ${ }^{61} \mathrm{Ihm}$ war bewusst, dass er seine neue Stellung der Protektion durch die Grafen der Neuensteiner Linie verdankte.

1756 zeigte sich, dass man im hohenlohischen Kernland keineswegs den im fernen Ohrdruf wirkenden Johann Andreas Bach vergessen hatte. Die Stelle des Organisten in Weikersheim war vakant und es gab sieben Bewerber. Der Weikersheimer Stadtmusiker Spindler brachte den Namen Bachs ins Spiel. Das Weikersheimer Konsistorium bescheinigte Johann Andreas alle erforderliche Eigenschafften zu dem hiesigen Organisten Dienste. Dieser war aber in Ohrdruf vergleichsmäßig gut versorgt und hatte eine Besoldung gefordert, die sich Weikersheim bei weitem nicht leisten konnte. So kann man auf gedachten Bach weiter keine Rechnung machen, heißt es abschließend in der Akte des Konsistoriums. ${ }^{62}$ Den Zuschlag

56 La 35 Regierung II Bü 841 Qu 6: Brief Bachs vom 13.3.41 an seine Freundin.

57 La 35 Regierung II Bü 841 Qu 15.

58 Kirchensaller Taufbuch, Eintrag vom 31. Mai 1741. Hier sind die Eltern als verheiratet nachgewiesen, die Hochzeit in Gnadental muss kurz vorher erfolgt sein. Von einer späteren Hochzeit (1743 in Langenburg) gehen Frickel (wie Anm. 3), S. 45, Freyse (wie Anm. 4), S. 71 und noch Caesar (wie Anm. 3), S. 77 aus, das erste Kind ist Freyse unbekannt. Die Langenburger Affäre des Johann Andreas ist in der Literatur noch weitgehend unbekannt.

59 La 35 Regierung II Bü 841 Qu 20.

60 La 10 P 4, GL 35 Bü 609.

61 La 10 P 5 Qu 1, La 35 Regierung II Bü 841 Qu 50: Brief vom 4. November 1743 (Zitat).

62 Zitate We 41 Schubl. 36 Fasz 165 Qu 4. 
erhielt Franz Vollrath Buttstedt aus Erfurt. GrafCarl Ludwig von Hohenlohe-Weikersheim hatte dies kurz vor seinem Tod noch entschieden.

1766 wurde Johann Andreas Bach zum Präzeptor der 5. Klasse bestellt. ${ }^{63} 1779$ starb er im Alter von 66 Jahren. ${ }^{64}$ Über zwei Jahrzehnte hat er an beiden Ohrdrufer Orgeln als Organist gewirkt und er hat in seiner Heimatstadt Lehrerdienste geleistet. Sein Lebensweg blieb aber keineswegs auf Ohrdruf beschränkt. Die Zeit als Tafeldecker in Langenburg wäre vielleicht unter anderen Umständen Ausgangspunkt einer Karriere an der Langenburger Residenz geworden. Es ist ein Capriccio über den Namen Bach von ihm bekannt, Johann Andreas besaß zudem zahlreiche Notenhandschriften, vor allem solche seines Onkels Johann Sebastian Bach. ${ }^{65}$

Die Ohrdrufer Neffen des Johann Sebastian Bach knüpften mit Hohenlohe - mit der Region wie mit dem Adelshaus - enge Verbindungen. Die Zahl ihrer Kinder ist zu groß, um auf die nächste Generation detailliert einzugehen. Die Orgel in Ohrdruf blieb bis 1814 unangefochten in der Hand der Nachfahren des Johann Christoph Bach. Der letzte war Johann Christoph Georg Bach (1747-1814), ein Sohn des Johann Andreas Bach. 1779 wurde er Organist an der Michaeliskirche zu Ohrdruf als Nachfolger seines Vaters. Auch weitere Orgeln in der thüringischen Grafschaft Obergleichen wurden in den folgenden Generationen mit Mitgliedern der Familie Bach besetzt. Sie übernahmen in der Grafschaft Kantorate, Lehrerstellen, darunter auch leitende, sowie Pfarrerstellen. ${ }^{66}$ Zuständig waren für sie immer das Haus Hohenlohe und seine Behörden in Thüringen und Franken.

\section{Bach in Langenburg}

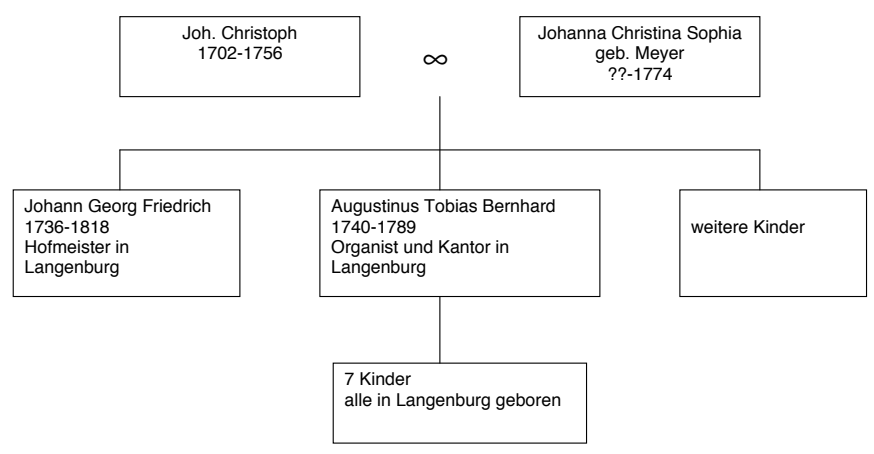

63 GL 35 Bü 650 und La 10 AA 30/2.

$64 \mathrm{La} 10 \mathrm{CC} 34 / 1$.

65 Schulze (wie Anm. 12), S. 40-41 über das Capriccio. Johann Andreas Bach war Nachbesitzer des von seinem Vater angelegten „Andreas-Bach-Buches“ und der „Möllerschen Handschrift“, die etwa zu einem Viertel Frühwerke Johann Sebastian Bachs enthielten.

66 Artikel Johann Christoph Georg Bach (83). In: MGG Personenteil I, 1999, Sp. 1299. GL 35 Bü 670, GL 35 Bü 719. La 10 CC 34/3. La 10 EE 13. GL 35 Bü 688. - Zu den jüngeren Generationen der Bachfamilie auch allgemein Caesar (wie Anm. 3), S. 78. 


\section{Die Brüder Augustinus Tobias Bernhard (1740-1789) und Johann Georg Friedrich (1736-1818) in Langenburg}

Zwei Großneffen des Johann Sebastian Bach müssen noch behandelt werden: die Brüder Augustinus Tobias Bernhard und Johann Georg Friedrich - in Langenburg tätige Mitglieder der Musikerfamilie. Sie waren Söhne des Johann Christoph Bach (des jüngeren), der die Tochter des langenburgischen Kanzleisekretärs geheiratet hatte. Dadurch bestanden familiäre Verbindungen des Ohrdrufers nach Langenburg.

In Langenburg wurde 1764 die Orgel der Stadtkirche gründlich erneuert. Fünf Jahre später wurde Augustinus Tobias Bernhard als Präzeptor und Kantor eingestellt. Er war damals candidatus juris. ${ }^{67}$ Ein 1769 abgefasstes Verzeichnis über die Musikalien und Musikinstrumente, die dem Präzeptor Bach zur Verfügung standen, ist sehr aufschlussreich und verdeutlicht die Verwobenheit von Kirchen- und Hofmusik in Langenburg. Demnach verfügte er über fünf Oboen, zwei Fagotte, vier Flöten (Flutes dolces), zwei Alt-Flöten, vier Traversflöten, sechs Hörner, zwei Trompeten, fünf Violinen, zwei Violen, vier Violoncelli und einen Bass. Der Verfasser des Verzeichnisses erklärt am Schluss, dass er nur für das Vorhandensein einiger weniger Instrumente persönlich garantieren kann, vor die übrigen Nrn. aber kann ich nicht haften, weil sie wegen ordin[airen] und extraordinairen Music in Schloss bald in diese, bald in jene Hand kommen. Mit den genannten Instrumenten wurde also auch am Langenburger Hof musiziert. Bei einigen Instrumenten ist der Aufenthalt genauer angegeben: Lakai Hebeisen hatte eine der Oboen. Ein Fagott, ebenso ein Horn, eine Trompete, zwei Violen und ein Violoncello befanden sich in der Lakaien-Stube. Der Registrator Duncker und der Pfeifer Spreng hatten jeweils eine Traversflöte in Besitz, Spreng zusätzlich noch ein Violoncello. Eine Violine befand sich ohne nähere Angabe im Schloss, über eine weitere verfügte der Lakai Hebeisen, weitere befanden sich in den Händen von Kindern, die nicht unbedingt im Schloss untergebracht waren. ${ }^{68}$ Die Instrumentenausleihe zeigt, dass die Diener und andere Hofbedienstete zum Musizieren herangezogen wurden. Und die Zahl der dorthin ausgeliehenen Instrumente legt nahe, dass die Musik am Hof die eigentliche Tätigkeit Bachs werden würde.

1770 hat Bach die Tochter des Hofverwalters Helmschmidt aus Langenburg geheiratet. ${ }^{69}$ Er war also wie sein Öhringer Onkel Organist an einer neuen Orgel. Die Sorge um diese Orgel, die erst 1783 zur vollen Zufriedenheit Bachs hergestellt

67 La 35 Bü 769: Deutsche und lateinische Schule zu Langenburg, 1726-1789, Qu 184: Protokoll über die Verpflichtung des neuen Präzeptors Bachen am 15. November 1769.

68 Sturz von 1769 La 35 Bü 775 Schule zu Langenburg, u. a. Schulvisitationen, Stürze über Musikinstrumente und Noten, Qu 23 und 23a Stürze über Noten und Instrumente in Langenburg unter Präzeptor Bach.

69 Freyse (wie Anm. 4), S. 50-52 (fälschlich Helmschrot); Caesar (wie Anm. 3), S. 75. 
war, dominierte die Amtszeit. ${ }^{70}$ Auch in seiner Funktion als Kantor gestaltete er das Musikleben in der hohenlohischen Residenz. Zwanzig Jahre, bis zu seinem Tod 1789, wirkte er in Langenburg. Augustinus Tobias Bernhard Bach hinterließ Kinder, die aber nicht mehr Musiker waren und die Hohenlohe verließen. Das Wissen über den Bruder Johann Georg Friedrich ist spärlicher. ${ }^{71}$ Immerhin erhielt er die Stellung des Hofmeisters in Langenburg. Zu seinen Aufgaben gehörte die Erziehung der Kinder des Grafen. Hier zeigt sich wieder eine enge vertrauliche Verbindung zwischen der Musikerfamilie Bach und dem - inzwischen - Fürstenhaus Hohenlohe. Johann Georg Friedrich blieb Junggeselle, wurde aber relativ alt.

\section{Resümee}

Das Wirken der Musikerfamilie Bach in Hohenlohe wurde über mehrere Generationen dargelegt. Die sich ausbreitende Musikerfamilie sickerte um 1700 allmählich auch in Hohenlohe ein, und zwar sowohl ins thüringische Ohrdruf wie ins fränkische Weikersheim. Bis Ende des 18. Jahrhunderts hinein lassen sich musikalisch engagierte Familienmitglieder in Hohenlohe finden. Am spektakulärsten freilich waren die Aufenthalte von Bach-Neffen in Langenburg und Öhringen: Johann Andreas Bach wirkte von 1737 bis 1741 am Hof in Langenburg, bis er die Residenz wegen eines Vorfalls überraschend verlassen musste, und sein Bruder Johann Heinrich Bach von 1735 bis 1783 in der gemeinschaftlich-hohenlohischen Stadt Öhringen, u. a. als Organist an der Stiftskirche. Ihre Aufenthalte in Hohenlohe verwundern aber nicht, sondern stehen im Kontext einer Vielzahl von Verbindungen der Musikerfamilie mit Hohenlohe, aus der sie nur herausragten. Bemerkenswert sind außerdem die Aufenthalte zweier Neffen Johann Sebastian Bachs in Weikersheim - Johann Christian und Johann Georg sowie später zweier Großneffen - Augustinus Tobias Bernhard und Johann Georg Friedrich - in Langenburg.

Die Hohenlohe förderten die Musikerfamilie Bach und protegierten sie bei Stellenbesetzungen. Der Ohrdrufer Zweig, also die Nachkommenschaft des Johann Christoph Bach, Johann Sebastian Bachs älterer Bruder, kam so auch im fränkischen Hohenlohe zum Zuge. Das hohenlohische Ohrdruf war allerdings der bevorzugte Wirkungskreis, worauf sich die Förderung durch die Hohenlohe auch konzentrierte.

70 La 35 Bü 767 Überlegungen über die Anschaffung einer neuen Orgel in der Stadtkirche und Reparatur der Orgel, 1763-1769, 1783. La 35 Bü 767 Qu 3: A.T. Bach an das Konsistorium Langenburg vom 21. Sept. 1783: Reparatur der Orgel sei abgeschlossen, fordert zur Zahlung der im Akkord vereinbarten Summe auf.

71 Freyse (wie Anm. 4), S. 44. 
Lehrer und Organist war die typische Kombination. Bezahlte Organistenstellen standen selten zur Verfügung, daher wurde man Lehrer, um so auch musikalisch wirken zu können. Die genannten Mitglieder der Familie Bach waren keine Vollmusiker und Genies wie Johann Sebastian Bach oder seine Söhne. Aber auch sie waren engagierte Musiker. Ihre Arbeit geschah eher „im Kleinen“: Typisch sind Orgelspiel beim Gottesdienst, Organisation des Kurrendesingens, Choraufführungen, teilweise auch Durchführung der Hofmusik und manchmal auch Komponieren. Und zuweilen standen sie für die Feste am Hof organisatorisch und komponierend zur Verfügung. 